\title{
Atomic Spectroscopy N \\ Three-dimensional Analyses of Geological Materials on Nanoscale by Electron Tomography
}

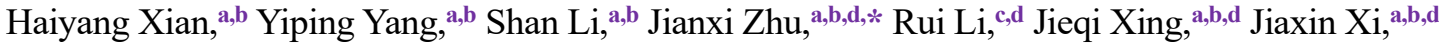 \\ Hongmei Yang, ${ }^{\text {a,b }}$ Xiaoju Lin, ${ }^{\text {a,b }}$ Xiao Wu, ${ }^{\text {a,b }}$ and Hongping $\mathrm{He}^{\mathrm{a}, \mathrm{b}, \mathrm{d}}$ \\ ${ }^{a}$ CAS Key Laboratory of Mineralogy and Metallogeny/Guangdong Provincial Key Laboratory of Mineral Physics and Materials, Guangzhou Institute of \\ Geochemistry, Chinese Academy of Sciences (CAS), Guangzhou 510640, P.R. China \\ ${ }^{\text {b }}$ CAS Center for Excellence in Deep Earth Science, Guangzhou 510640, P.R. China \\ ${ }^{\mathrm{c}}$ Center for Lunar and Planetary Sciences, Institute of Geochemistry, Chinese Academy of Sciences, Guiyang 550081, P.R. China \\ ${ }^{\mathrm{d}}$ University of Chinese Academy of Sciences, Beijing 100049, P.R. China
}

Received: December 01, 2021; Revised: February 15, 2022; Accepted: February 16, 2022; Available online: February 17, 2022.

DOI: $10.46770 / A S .2022 .012$

ABSTRACT: Electron tomography (ET), an electron-microscopy-based technique that provides three-dimensional (3D) structural information from a tilt series of two-dimensional (2D) projections, has promoted the in-depth investigation of biological molecules in structural biology and the analysis of material structures on the atomic scale in physical sciences. Although ET has developed rapidly as an effective technique wherein atomic-scale resolution has been achieved by using advanced transmission electron microscopy systems, it has not been widely used by the earth and planetary science community. Herein, we verify the applicability of ET in research related to earth and planetary science. The data demonstrate that ET can be used to observe the 3D morphology of mineral grains and 3D distributions of the chemical components of earth and planetary materials. Notably, ET coupled with spectroscopy, including electron energy loss spectroscopy and energy dispersive spectroscopy, is an effective technique for studying the 3D distribution of elements and their various oxidation states in geological materials. Atomic ET is also a promising technique for detecting trace elements in host minerals. The use of ET can advance the study of earth and planetary materials because it provides additional 3D information about geological objects.

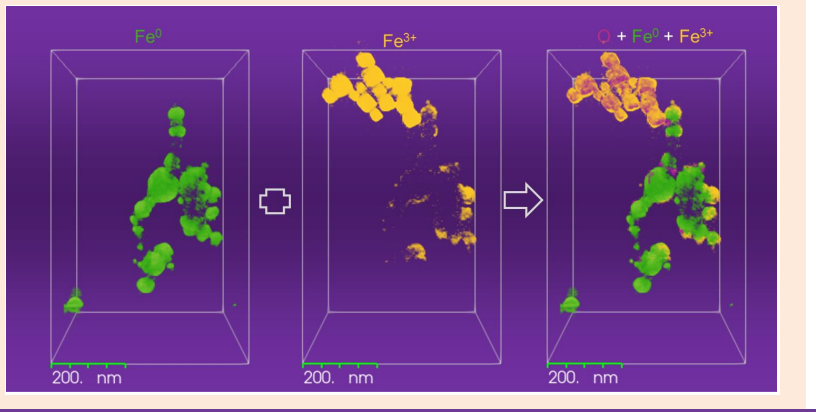

\section{INTRODUCTION}

Since the discovery of matter waves by Louis de Broglie in $1924,{ }^{1}$ the wave properties of electrons have been exploited to image matter from the micro to nanoscale. This has been achieved by a technique called electron microscopy (EM) that includes transmission electron microscopy (TEM), scanning electron microscopy (SEM), and scanning transmission electron microscopy (STEM). These EM techniques enable twodimensional (2D) imaging of matter on the nanometer scale. However, 2D images are projections from inherently threedimensional (3D) samples. The missing third dimension is necessary to understand the functionality of many structures. ${ }^{2}$
Thus, 3D-reconstruction techniques have been developed from 2D projections of EM images.

The 3D-reconstruction technique is termed tomography, which means "imaging by slices". 3 The fundamental mathematics underlying tomography were established by Johan Radon in $1917 .{ }^{4}$ Thereafter, the tomography theory stagnated until 1956 when it was first applied in astronomy using a set of projections of the sun from the earth. ${ }^{5}$ Since the $1960 \mathrm{~s}$, various types of computed tomography (CT) techniques, e.g., X-ray $\mathrm{CT},{ }^{6-8}$ ultrasound $\mathrm{CT},{ }^{9}$ and atom probe tomography, ${ }^{10}$ have been developed for application toward the research related to both life and physical sciences. 
Electron tomography (ET) is a CT technique that is based on TEM. ET was first developed for use in the research associated with life sciences in the $1960 \mathrm{~s}^{2,11,12}$ and then adopted for research in materials science 20 years later. ${ }^{13}$ With advances in instrumentation, reconstruction algorithms, and computational power over the past few decades, ET has been successfully used to reconstruct both soft and hard materials with angstrom-level resolution. ${ }^{14}$ While ET is still a relatively new microscopy technique, it is one of the most useful tomographic tools in various fields. It continues to benefit from the advancement of new TEM techniques such as spherical aberration correctors and improved detector sensitivity. It has numerous advantages including high resolution (from nano to atomic scale level) and 3D imaging. On the contrary, the nanosized limits and electron beam damage of ET may restrict its application in some fields with complex samples. For instance, in earth and planetary science, inhomogeneous samples with multi-components may not be appropriately sampled; if various components of a sample have different sensitivity to electron radiation, it is even hard to obtain authentic images.

Although the first application of tomography was in astronomy, and ET has been well developed as an advanced technology with angstrom-level resolution using modern TEM systems, ${ }^{15}$ this technique is largely overlooked by the earth and planetary science community. Only a few recent studies have employed ET for research in the field of earth and planetary science (e.g., Refs. ${ }^{16-18}$ ). To facilitate wide-scale implementation of ET, in this study, we briefly introduce the basic concepts of ET and verify the applicability of ET in research pertaining to the field of earth and planetary science.

\section{BASIC CONCEPTS OF ELECTRON TOMOGRAPHY}

ET is a technique in which $3 \mathrm{D}$ structures of specimens are reconstructed from a series of 2D images acquired using TEM. As illustrated in Fig. 1, the ET process has two main components: acquisition of $2 \mathrm{D}$ images for a tilt series and reconstruction of a 3D model from the acquired 2D images using algorithms. Herein, we briefly introduce various imaging modes and several basic reconstruction algorithms that are commonly used in ET-based studies.

\section{D image acquisition}

Imaging modes. Conventional TEM instruments always operate in two modes: the TEM mode with a parallel coherent beam of electrons and the STEM mode with a focused beam of electrons. In the TEM mode, the incident electron beam interacts with the atoms in the specimen, and the exiting electron wave contains (a) Tilt Series Acquisition

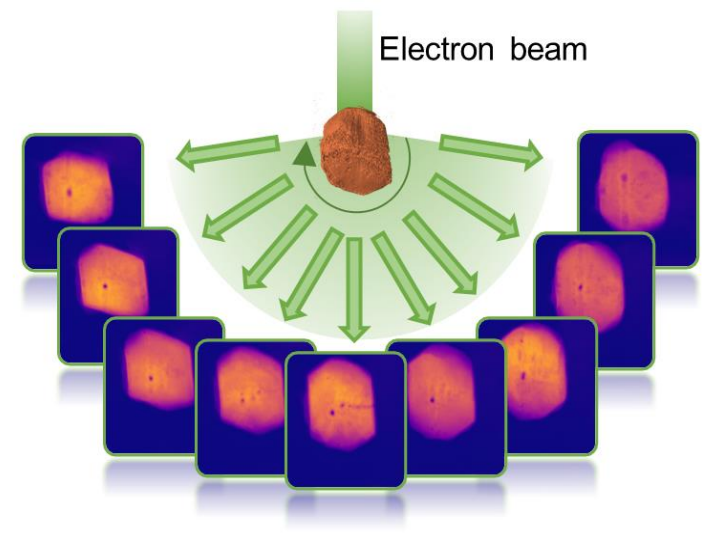

(b)

$$
\text { 3D Object Reconstruction }
$$

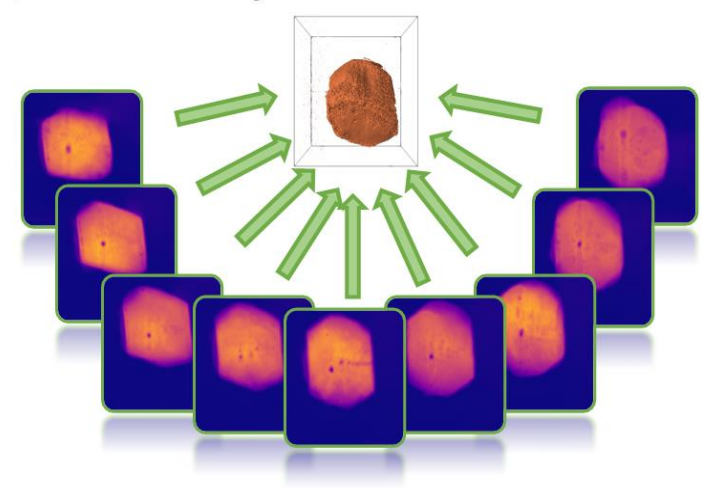

Fig. 1 Schematic of ET. Acquisition of a series of TEM images at different tilt angles (a) and reconstruction of the 3D structure of a specimen from the acquired tilt series (b).

information about the sample. The contrast in the TEM images is controlled by the density and thickness of the specimens and is affected by the crystallographic orientations at different tilt angles. Thus, TEM bright-field (BF) image tomography is used for the analysis of biological materials, with a negligible effect of oriented diffraction contrast. In addition, energy-filtered TEM can be employed for the 3D reconstruction of the chemical composition of samples. ${ }^{19}$

In the STEM mode, the high-angle annular dark-field (HAADF) image is used for effectively constructing ET images because the image intensity is mainly affected by the atomic number ( $Z$ ), which is positively correlated to the density of the atoms. However, beam damage is a considerable issue that limits the application of STEM for the analysis of electron-sensitive materials. However, this issue typically does not arise in most robust specimens in materials science. Furthermore, techniques involving the combination of STEM and spectroscopy, such as electron energy loss (EEL) spectroscopy (EELS) and energy dispersive spectroscopy (EDS), are effective for the 3D reconstruction of the chemical composition of samples. 


\section{Reconstruction of 3D model}

Direct Fourier reconstruction. A projection of an object at a given angle in real space can be achieved as a central section through the Fourier transform of the object. ${ }^{20}$ When a series of projections (central sections) is acquired, the 3D Fourier space of the object can be observed. Fourier inversion then allows the generation of a 3D model of the object. Such a reconstruction from the inverse Fourier transform is called a direct Fourier reconstruction. Inverse Fourier transform requires a continuously varying function; thus, direct Fourier reconstruction requires numerous images in the tilt series to ensure fidelity and accuracy. Furthermore, direct Fourier reconstruction requires significantly large computer memory. Thus, direct Fourier reconstruction is not as commonly used as the real-space back-projection methods described below.

Real space Back-projection. Radon transform maps a 3D object onto a 2D plane by calculating the line integrals (projections) of object density through all "projection rays" parallel to a given axis. If the density of a $3 \mathrm{D}$ object is projected onto $2 \mathrm{D}$ images at various tilt angles, the $2 \mathrm{D}$ images can be used to reconstruct the $3 \mathrm{D}$ object using an inverse Radon transform. The inverse Radon transform in real space is known as a back projection. As a requirement for real-space back projection, the intensity of the $2 \mathrm{D}$ images of the target object must be a monotonic function of the physical quantity to be reconstructed. ${ }^{21}$

Weighted Back-projection. Although back-projection does not require large computer memory, uneven sampling of spatial frequencies causes blurred features in reconstructed model. A weighting filter in the Fourier space is employed to correct the blurred features. Reconstruction using such a weighting filter is called weighted back-projection (WBP).

Iterative reconstruction. Although the WBP method is widely used in the ET community, there are still other artifacts in the reconstructed model. One simple way to correct these artifacts in the reconstructed model is to use original projections. The correction is performed by comparing the difference between the reprojections of the reconstructed model and the original projections. The difference can be minimized interactively. ${ }^{22}$ The two most commonly used interactive reconstruction algorithms are the algebraic reconstruction technique (ART) ${ }^{23}$ and the simultaneous iterative reconstruction technique (SIRT). ${ }^{22}$ ART compares the reconstruction with a single projection once, while SIRT compares all projections simultaneously. SIRT is more commonly used than ART because it tends to be more computationally stable than ART when images are noisy. ${ }^{22}$ SIRT requires a relatively large number of projections $\left(1^{\circ}-2^{\circ}\right.$ tilt interval and $\pm 70^{\circ}$ tilt angles). In addition to these conventional reconstruction methods, many advanced algorithms, such as the generalized Fourier iterative reconstruction (GENFIRE), ${ }^{24}$ and

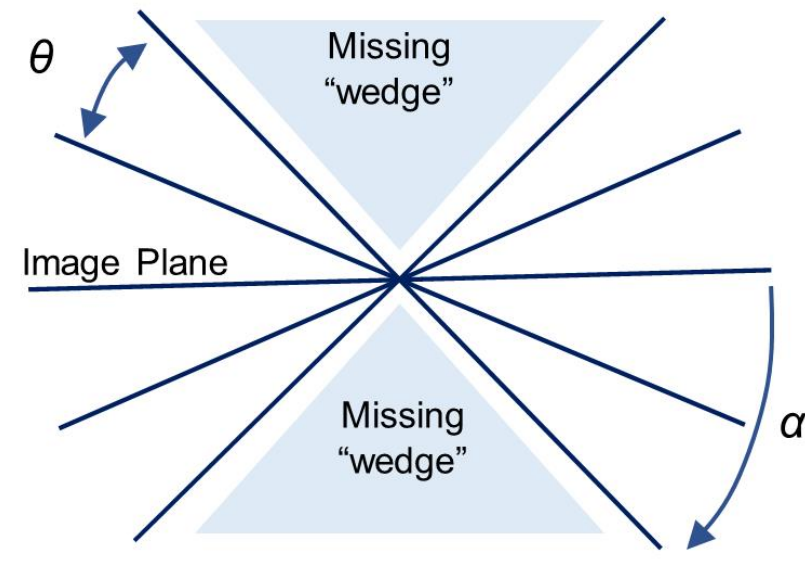

Fig. 2 Illustration of the missing wedge of information between the tilt angle $(\alpha)$ and $90^{\circ}$.

deep-learning-aided information recovery ${ }^{25}$ have been developed in recent years to improve the quality of reconstructed images.

\section{Missing wedge and resolution}

The resolution of ET is anisotropic because during image acquisition, the object structure is inherently under sampled. If the alignment of the tilt series is appropriate, the resolution along the tilt axis is equal to the original experimental resolution. The resolution along the image plane perpendicular to the tilt axis is reduced owing to the discrete number of projections $(N)$. The resolution $(d)$ can be estimated using an equation relating $N$ to the diameter of the reconstruction volume, $D .{ }^{26}$

$$
d=\pi D / N
$$

This equation assumes a full-range tilt series for the reconstruction. However, practical TEM experiments are always limited by the tilt angle ( $\alpha$ is always lower than $\pm 80^{\circ}$ ), leading to a missing "wedge," as illustrated in Fig. 2, although ET can be performed without a missing wedge in a few cases. ${ }^{27}$ The missing "wedge" further reduces the resolution along the projection axis by an elongation factor, $e:^{28}$

$$
e=\sqrt{\frac{\alpha+\sin \alpha \cos \alpha}{\alpha-\sin \alpha \cos \alpha}}
$$

The aforementioned resolutions along different directions are optimal, as the resolutions are always affected by other factors such as stage drift and image alignment. The effects of stage drift and image alignment are constrained by the stability of the TEM stage; thus, an optimized stable stage is necessary to perform ET experiments. 


\section{Electron Tomography Workflow}

\begin{tabular}{|c|c|}
\hline $1 \begin{array}{c}\text { Sample } \\
\text { Preparation }\end{array}$ & Powder, section or pillar by FIB \\
\hline 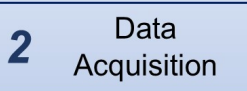 & $\begin{array}{l}\text { Manual- or auto-acquisition using } \\
\text { software (e.g., SerialEM) }\end{array}$ \\
\hline $\begin{array}{c}\text { Data } \\
3 \text { Alignment \& } \\
\text { Reconstruction }\end{array}$ & $\begin{array}{l}\text { Image alignment and 3D } \\
\text { reconstruction using software } \\
\text { (e.g., imod, TomoJ, and Tomviz) }\end{array}$ \\
\hline $\begin{array}{c}\text { Analyses \& } \\
\text { Visualization }\end{array}$ & $\begin{array}{l}\text { 3D Rendering using software } \\
\text { (e.g., FIJI and Tomviz) }\end{array}$ \\
\hline
\end{tabular}

Fig. 3 Workflow of ET experiment.

\section{EXPERIMENTAL WORKFLOW}

The workflow for ET consists of four steps (Fig. 3): sample preparation, data acquisition, data alignment and reconstruction, and analyses and visualization. Herein, several examples are presented to demonstrate how to perform ET experiments for various geological samples using conventional TEM.
Sample preparation. In earth and planetary science, either powder or bulk solid samples can be analyzed through ET. The samples used for the ET experiments should have a nanoscale size (e.g., $<100 \mathrm{~nm}$ at $200 \mathrm{keV}$ ) at least one-dimensional. Otherwise, a TEM system that is operated at a considerably high voltage (e.g., $1 \mathrm{MeV}$ ) is required. The samples for ET experiments are prepared by the method identical to that used for conventional TEM sample preparation; that is, powders can be directly dispersed on a TEM grid (e.g., 200-mesh copper grids with ultrathin carbon film are used in this study), whereas bulk solid samples can be thinned by either ion milling or focused ion beam (FIB) cutting.

Three samples were used for the ET experiments in this study. Two samples were in the powder state, while the third was a pillar sample cut by a FIB. A pillar FIB sample (Fig. 4) was cut from a pyrite section using a Thermo Fisher Scientific FEI Scios dualbeam system with a $\mathrm{Ga}^{+}$ion source (Institute of Geochemistry, CAS). One powder sample was simulated lunar soil (Figs. 5 and 6 ), while the other was a mixture of synthesized nanophase metallic iron (np- $\left.\mathrm{Fe}^{0}\right)$ and hematite $\left(\mathrm{Fe}_{2} \mathrm{O}_{3}\right)$ (Figs. 7-9). The simulated lunar soil (GIG-1) was prepared by ball milling a mixture of volcanic glass, basalt, synthesized np- $\mathrm{Fe}^{0}$, and minor monominerals. The volcanic glass (containing $52.82 \mathrm{wt} \% \mathrm{SiO}_{2}$, $14.92 \mathrm{wt} \% \mathrm{Al}_{2} \mathrm{O}_{3}, 9.10 \mathrm{wt} \% \mathrm{FeO}$, and $7.52 \mathrm{wt} \% \mathrm{MgO}$ ) and basalt (containing $49.28 \mathrm{wt} \% \mathrm{SiO}_{2}, 17.22 \mathrm{wt} \% \mathrm{Al}_{2} \mathrm{O}_{3}, 8.14 \mathrm{wt} \% \mathrm{FeO}$,
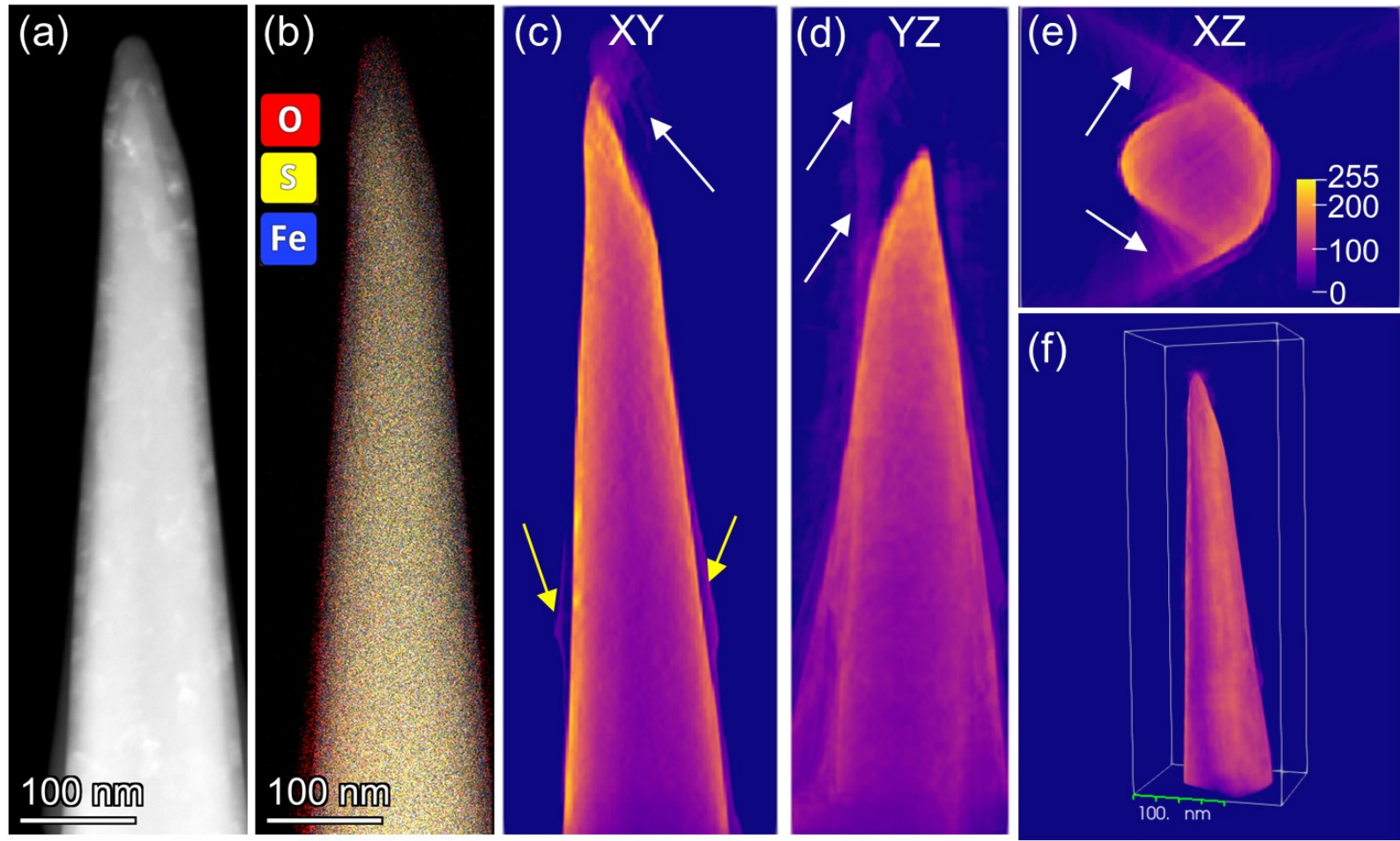

Fig. 4 HAADF-STEM image (a) and STEM-EDS elemental map (b) of a pyrite pillar sample prepared using FIB cutting; and WBP-reconstructed results $(\mathrm{c}-\mathrm{f})$ from tilt series acquired from $-63^{\circ}$ to $+70^{\circ}$ with interval of $0.5^{\circ}$. The white arrows in the slices indicate artifacts from reconstruction, while the yellow arrows indicate carbon contamination at the base of the needle. 


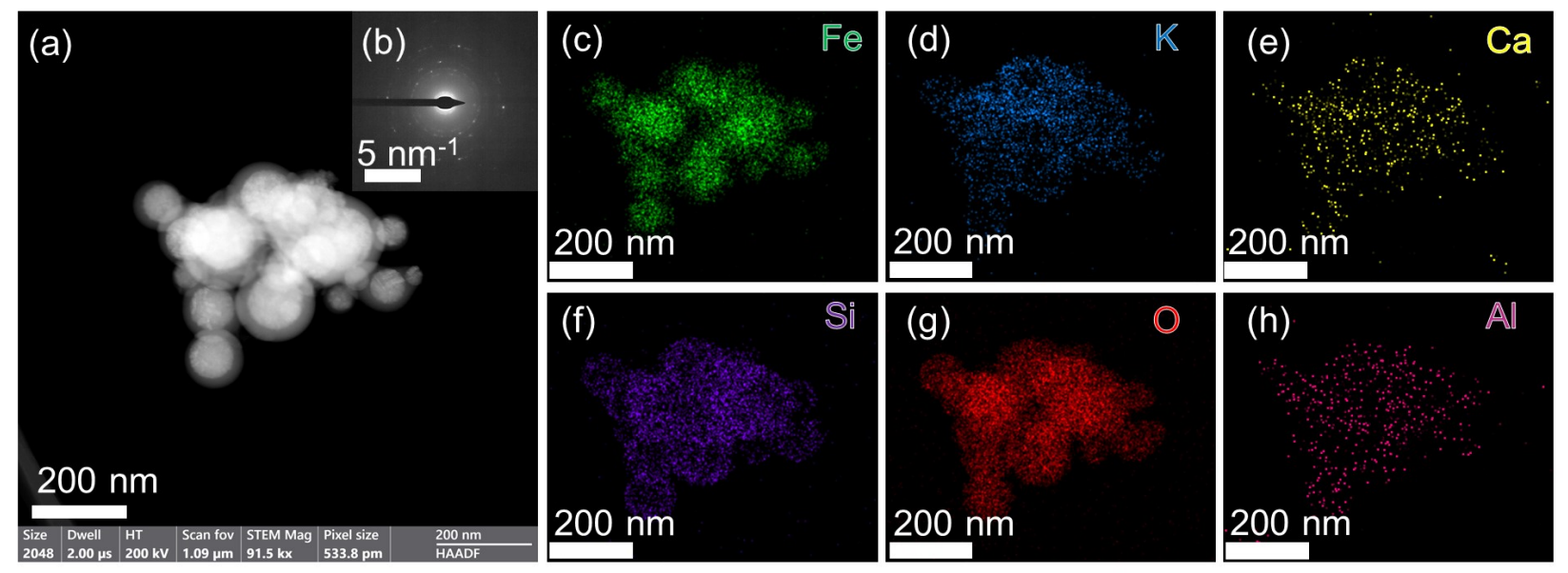

Fig. 5 HAADF-STEM image (a), SAED pattern (b), and EDS elemental maps (c-h) of an aggregate of nano-iron spheres from simulated lunar soil.
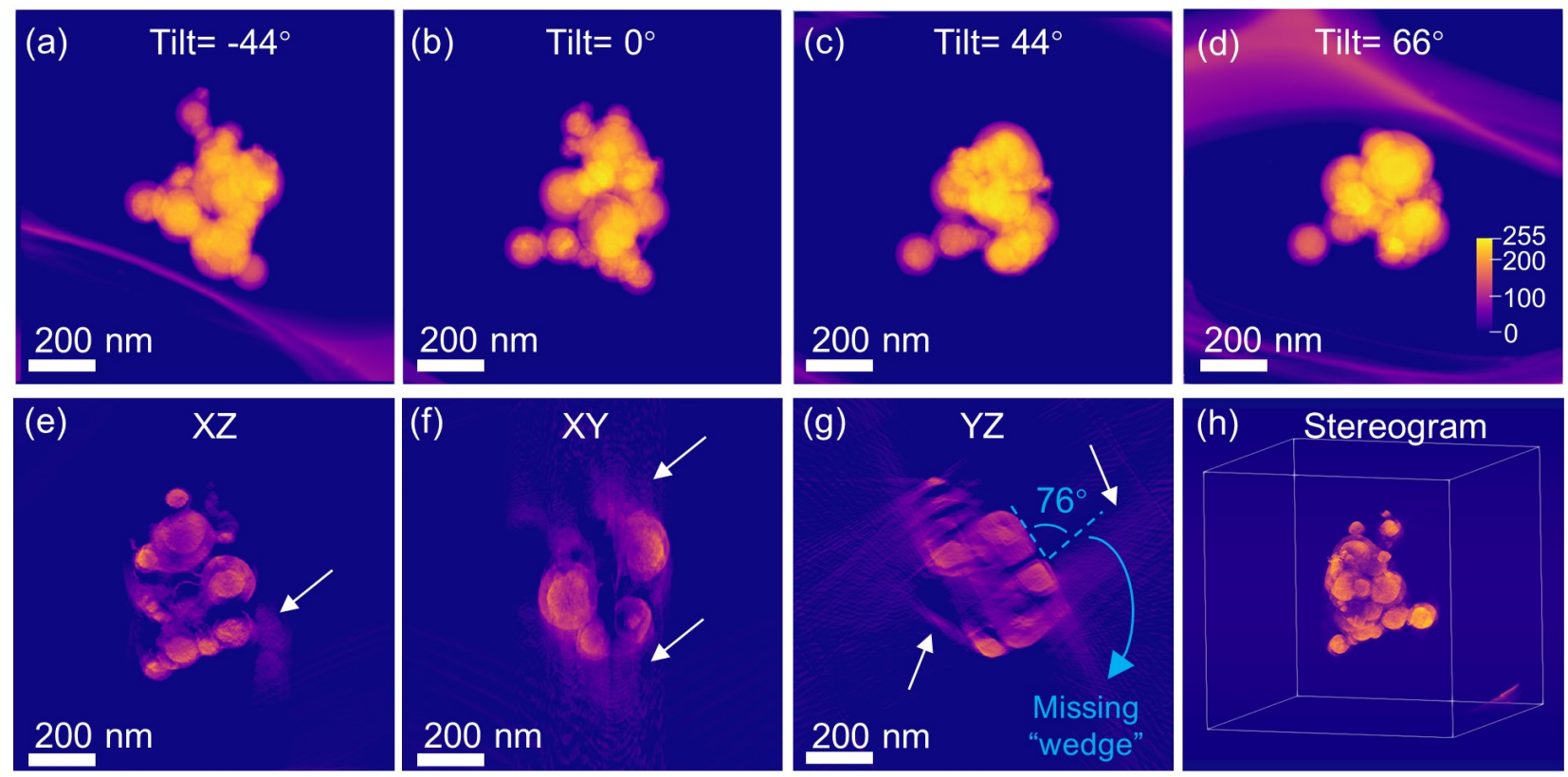

Fig. 6 Original HAADF-STEM tilt serial images acquired at $-44^{\circ}$ (a), $0^{\circ}(\mathrm{b}),+44^{\circ}$ (c), and $+66^{\circ}$ (d) with the rotation axis along the horizontal line in the image plane; representative slices in different directions form orthogonal view $(\mathrm{e}-\mathrm{g})$ and volume stereogram $(\mathrm{h})$ of the WBP-reconstructed 3D model from the tilt series acquired from $-44^{\circ}$ to $+66^{\circ}$ with interval of $2^{\circ}$. The white arrows in the slices indicate artifacts from reconstruction.

and $5.43 \mathrm{wt} \% \mathrm{MgO}$ ) were collected from Dayizi volcano, Jilin, China.

Data acquisition. Data acquisition for ET requires a high-angle tilt stage in the TEM system. However, the small space between the sample holder and pole shoe in the TEM column limits stage tilting. For instance, the conventional single-tilt holder for the Thermo Fisher Scientific FEI Talos F200S TEM system used in this study can achieve a tilt angle of $\pm 40^{\circ}$, even though the stage is designed to acquire a tilt angle of $\pm 90^{\circ}$. Thus, special sample holders that facilitate the achievement of high tilt angles are required for data acquisition in ET. Herein, either Fischione cryotransfer tomography holder 2250 or a special tomography holder made by CHIP-NOVA was used on a Thermo Fisher Scientific Talos F200S TEM system operated at $200 \mathrm{kV}$ (Guangzhou Institute of Geochemistry, CAS). Three sets of tilt series were acquired in the STEM mode with different signals (e.g., HAADF and EELS).

The manual acquisition of a set of tilt series makes it difficult to do reduplicative TEM operations (e.g., tilting angle, focusing, and moving stage). ET data can be automatically acquired by using 

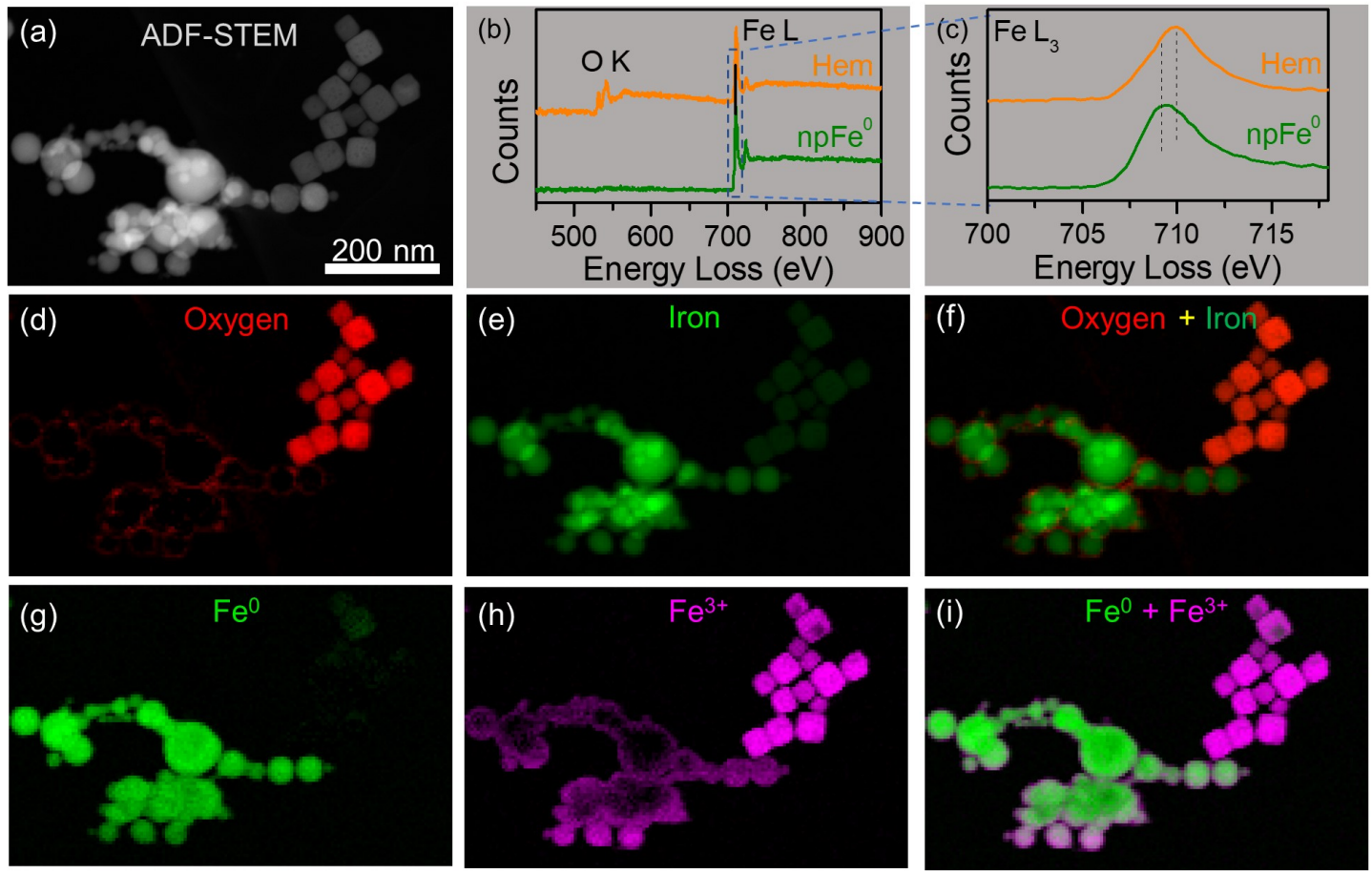

Fig. 7 HAADF-STEM image (a), EEL spectra ( $b$ and c), elemental maps $(d-f)$, and oxidation state maps ( $g-i)$ of a mixture of nano-iron spheres and hematite nanocrystals, with a tilt angle of $0^{\circ}$.

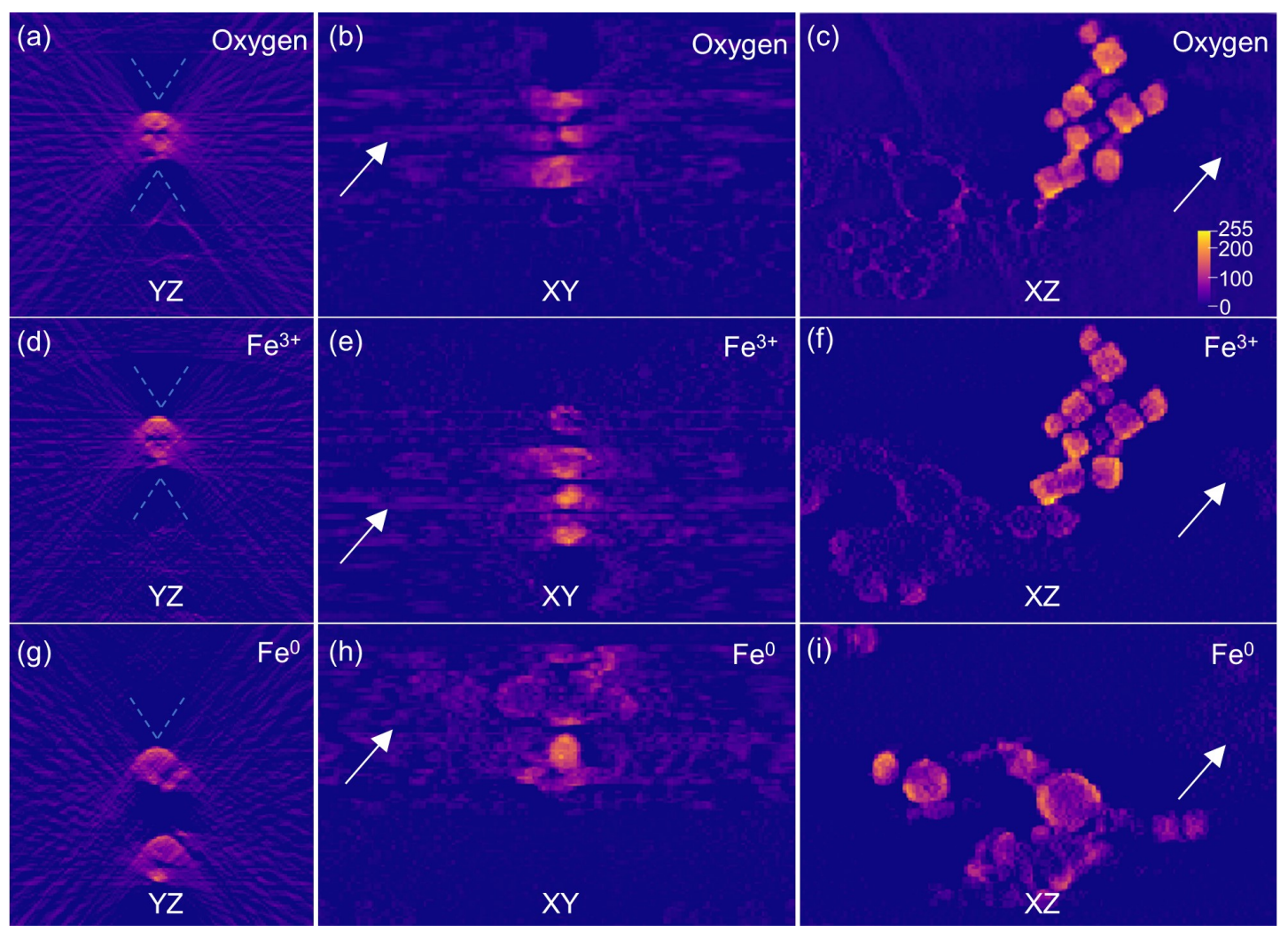

Fig. 8 Representative slices at different directions for WBP-reconstructed elemental data and oxidation states of the same specimen presented in Fig. 7. The results were reconstructed from a tilt series acquired at a maximum tilt angle of $\pm 54^{\circ}$ and with interval of $6^{\circ}$. 


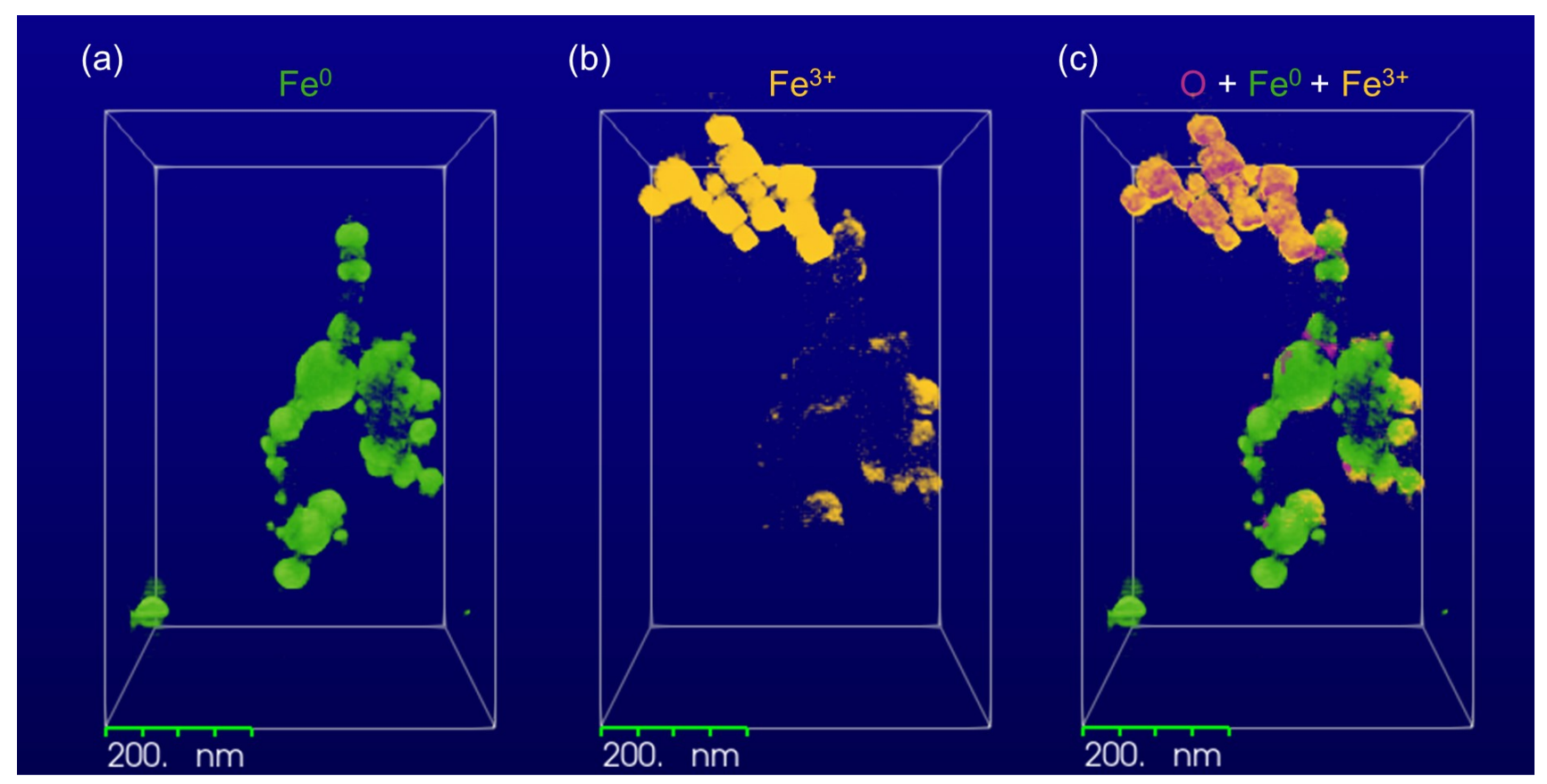

Fig. 93D views of the reconstructed models of elements and oxidation states for the same specimen presented in Figs. 7 and 8.

computer software. Automated acquisition of the ET tilt series using software is an advanced technique. Both academic (e.g., Leginon MSI-tomography ${ }^{29}$ and SerialEM ${ }^{30}$ ) and commercial (e.g., TEMography Recorder and Thermo Scientific Tomography) software are currently available. ${ }^{31}$ Herein, SerialEM was used to acquire the HAADF-STEM tilt series, whereas the STEM-EELS tilt series was acquired manually.

Data pretreatment, reconstruction, analysis, and visualization. The data should be pretreated before the reconstruction of the 3D model from the acquired tilt series (e.g., data format conversion) to match various reconstruction codes or software. For instance, in this study, the acquired 32-bit tilt serial HAADF-STEM $(2048 \times$ $2048)$ and EEL spectroscopic images $(142 \times 90)$ were converted to 16-bit images for further processing. The data should also be aligned because the region of interest is not always at the center of the images at different tilt angles in a practical ET experiment, which significantly affects the quality of the reconstructed $3 \mathrm{D}$ model. After image alignment, the tilt series can be reconstructed using algorithms (as described above) embedded in the reconstruction codes and software. The reconstructed model can then be visualized using computer software. There are many academic (e.g., FIJI, ${ }^{32}$ Tomviz, ${ }^{33}$ and $\operatorname{Imod}^{34}$ ) and commercial software (e.g., Avizo) to fulfill some of these requirements. Thus, the combined usage of various software provides a simple solution for accomplishing reconstruction of ET. In this study, FIJI was employed as a data pretreatment tool, Tomviz and Imod as reconstruction tools, and Tomviz as a visualization tool. The WBP algorithm was employed for all reconstructions presented herein.

\section{THEORETICAL SIMULATION}

Atomic ET (AET) is still under development although conventional TEM has been advanced to achieve atomic-scale resolution. Recent cutting-edge studies show that ET can achieve atomic-scale resolution and, thus, can be applied for the analysis of pure materials. ${ }^{35,}{ }^{36}$ However, AET of minerals containing impurities via conventional TEM is still challenging. To estimate the applicability of AET in the field of earth and planetary science research, theoretical simulation was conducted herein rather than a practical physical experiment; gold-bearing pyrite was used as the target specimen, wherein the occurrence of gold is in debate. ${ }^{37}$, ${ }^{38}$ A cubic pyrite model with $6 \times 6 \times 6$ supercells $(\sim 3 \mathrm{~nm}$ side length $)$ was constructed (Fig. 10). Based on the pure pyrite model, three iron atoms were randomly chosen to be substituted with gold atoms (Fig. 11), which is considered to be a gold-bearing pyrite model. The tilt series of the HAADF-STEM images of both models were simulated using a software based on the kinematicscattering method developed by $\mathrm{He}$ and co-workers. ${ }^{39}$ These simulated images were directly input into the reconstruction software without any pretreatment.

\section{RESULTS AND DISCUSSION}

Morphological electron tomography. One basic function of ET is to analyze the $3 \mathrm{D}$ morphology of specimens on the nanoscale. 

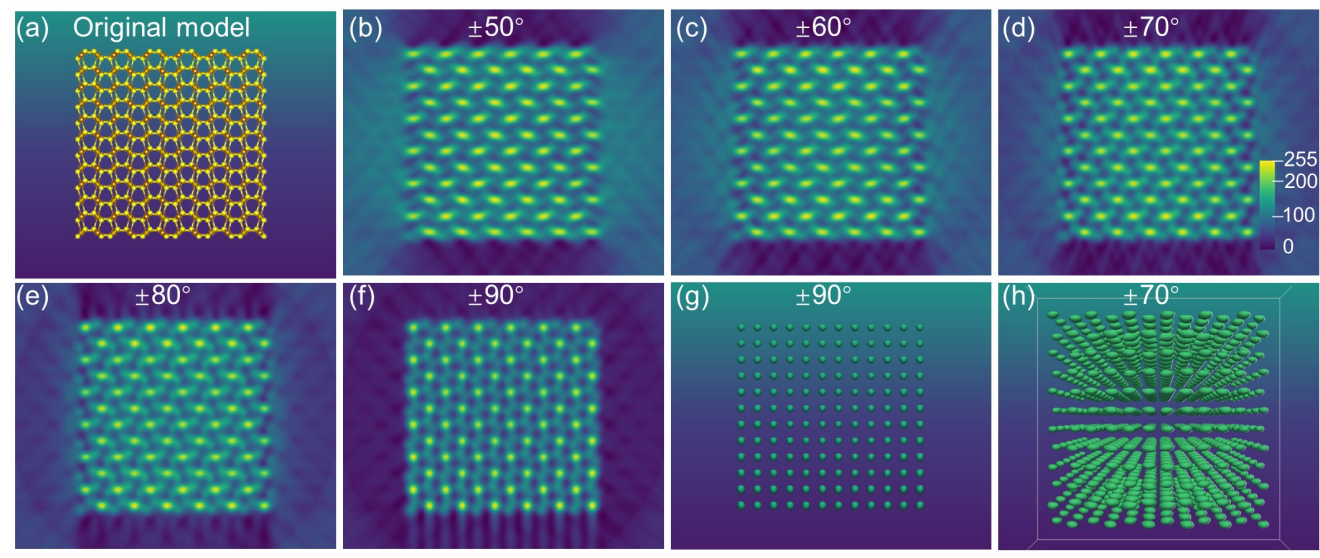

Fig. 10 Theoretical AET results for pure pyrite nanoparticle $(\sim 3 \mathrm{~nm})$. Original model (a); WBP-reconstructed slices along the rotation axis $(b-f)$; contour models in perspective and orthographic views ( $\mathrm{g}$ and $\mathrm{h}$ ). Yellow and brown spheres shown in (a) denote sulfur and iron atoms, respectively.
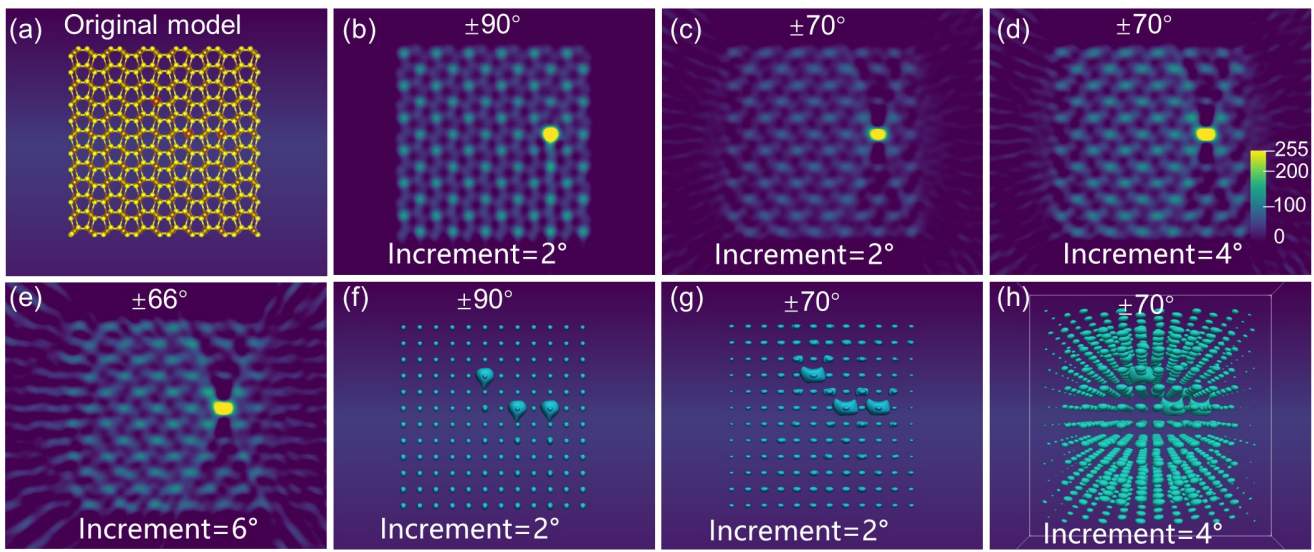

Fig. 11 Theoretical AET results for gold-bearing pyrite nanoparticle $(\sim 3 \mathrm{~nm})$. Original model (a); WBP-reconstructed slices along the rotation axis (b-e); and contour models in perspective and orthographic views ( $\mathrm{f}-\mathrm{h})$. Yellow, brown, and red spheres shown in (a) denote sulfur, iron, and gold atoms, respectively.

The WBP method is widely used in morphological electron tomography (MET), as described above; therefore, incoherent electron-scattering images (for which the contrast can be considered as a line integral of object density) must be employed in MET experiments. The HAADF-STEM technique, which produces typical electron-scattering images, was used to perform MET experiments in this study.

The MET experiment was first performed on a pyrite pillar prepared using a FIB. Before the acquisition of the tilt series, STEM-EDS maps of the sample were obtained. The results (Figs. $4 a-4 b)$ show some signals of oxygen dispersed on the surface of the pyrite pillar, suggesting that the pyrite sample was oxidized during transfer from the FIB chamber to the TEM system. The HAADF-STEM tilt series was acquired for the pyrite pillar over the scan range of $-63^{\circ}-+70^{\circ}$ with interval of $0.5^{\circ}$. The WBPreconstructed results (Figs. $4 \mathrm{c}-4 \mathrm{f}$ ) show that some artifacts (indicated by arrows) still existed in the reconstructed slices even when narrow interval of $0.5^{\circ}$ were used. The missing-"wedge"induced artifacts are possibly the largest, as shown in Fig. 4e. The acquired data were resampled to generate a new tilt series with interval of $2^{\circ}$. The results are almost the same as those shown in Figs $4 \mathrm{c}-4 \mathrm{f}$, indicating that the use of very narrow interval (e.g., $0.5^{\circ}$ ) is unnecessary for MET experiments. Despite the presence of these artifacts in the reconstructed slices, the 3D morphology of the measured pyrite pillar was accurately presented. The successful reconstruction of the pyrite pillar suggests that the WBP method is sufficiently accurate for reconstructing the $3 \mathrm{D}$ morphology of geological materials on the nanoscale. The edges of the pillar sample appeared to be much brighter (contrast) than those of the other parts. Although the edges of the pyrite pillar were oxidized, the observation of bright edges is not attributed to the presence of the oxidized surface because the oxidation did not introduce any heavy atoms. This may be because the center of the sample is too thick to allow the transmission of $200 \mathrm{keV}$ electrons, as the bright edges correspond to the thinner parts (edges) of the sample. Carbon contamination was also observed at the base of the pillar (yellow arrows in Fig. 4c), as confirmed by EDS analysis; this carbon accumulated during the acquisition of the tilt series. Carbon may originate from the Pt-based reactive gas used during 

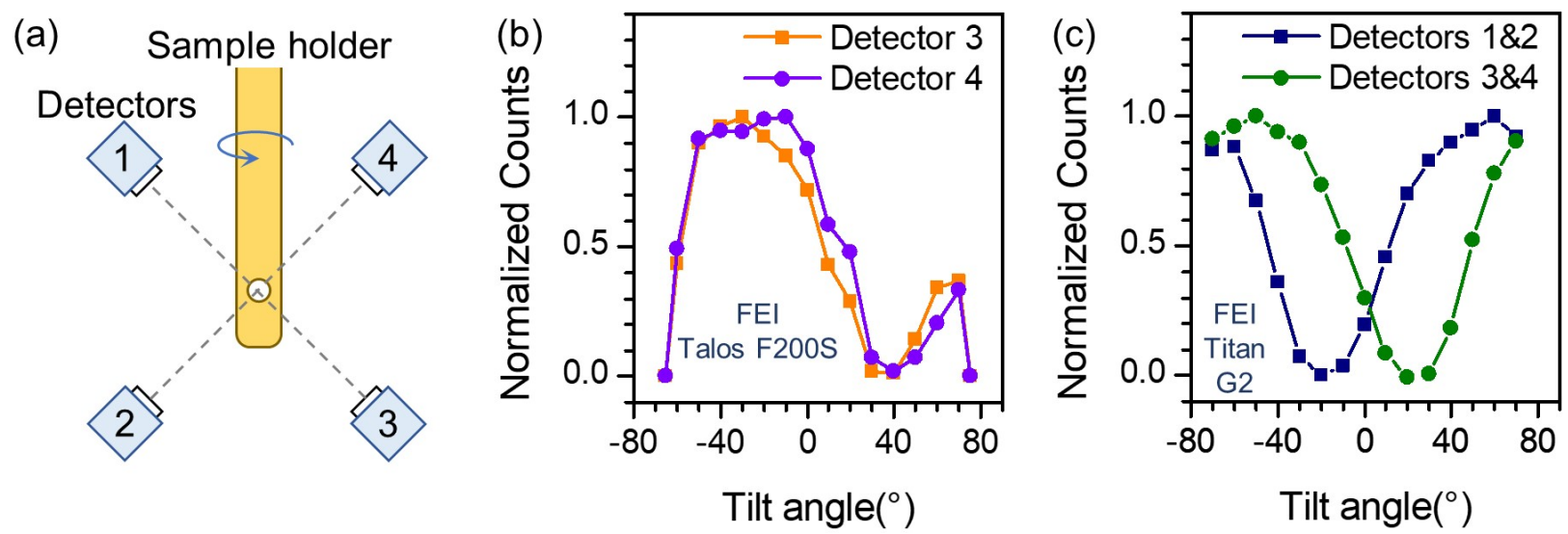

Fig. 12 Illustration of the geometry of the Super-X EDS detectors in the FEI TEM (a); Fe K $\alpha$ X-ray counts (7.1 keV) at each detector as a function of tilt angle, taken from EDS spectra acquired for the same hematite nanoparticle using an acquisition time of $5 \mathrm{~s}$ on an FEI Talos F200S TEM (b); backgroundsubtracted $\mathrm{Au} L_{\alpha} \mathrm{X}$-ray counts $\left(9.7 \mathrm{keV}\right.$ ) at each pair of detectors as a function of tilt angle on an FEI Titan G2, the data were cited from the literature ${ }^{42}$ (c).

the FIB sample preparation processes. ${ }^{40}$ Therefore, the region in the FIB sample for MET experiments should be far from the Pt deposition areas.

Aggregated particles from simulated lunar soil were then examined. The HAADF-STEM image (Fig. 5a) shows that the aggregate consists of nanospheres with a core-shell structure. By using a combination of selected area electron diffraction (SAED) analysis (image shown in Fig. 5b) with EDS-mapping (images shown in Figs. $5 \mathrm{c}-5 \mathrm{~h}$ ), the aggregates were identified as nano-iron spheres with an amorphous silicate coating.

A tilt series of the nano-iron sphere aggregates was acquired from $-44^{\circ}$ to $+66^{\circ}$ because the tilt angle was limited by the position of the sample on the TEM stage. The original HAADFSTEM images at different tilt angles (Figs. 6a-6d) show that the projections of the aggregate at different tilt angles have different contours. The image intensity (brightness) was higher where at the iron spheres overlapped, indicating that the intensity of the acquired images can represent the projected thickness or density of the nano-iron spheres. Therefore, the WBP method was used to reconstruct a $3 \mathrm{D}$ model of the aggregate. The results show that the shapes of the nano-iron spheres can be easily identified in different slices although some artifacts were observed in the slices in the orthogonal view (Figs. 6e-6g). The major artifacts on the YZ slices must be ascribed to the missing wedge because the angle between the two groups of artifacts was equal to the missing wedge angle in this ET experiment. These artifacts distort the shape from spherical to spindle. Minor artifacts along other directions did not change the spherical shape and may be caused by other factors (e.g., alignment). Although the artifacts cause distortions in the $\mathrm{YZ}$ plane, the 3D view of the reconstructed model (Fig. 6h) can reproduce the 3D spatial relations of these nano-iron spheres.

Spectroscopic electron tomography. EDS and EELS are two spectroscopic techniques that are commonly used in conventional TEM. Both these techniques can be employed for spectroscopic electron tomography (SET) for reconstructing the 3D distribution of elements and indicating their oxidation states. ${ }^{41}$ STEM-EDS tomography is mainly constrained by the detector position in the TEM column. ${ }^{42}$ Fig. 12a illustrates the geometry of the Super-X detectors in FEI TEM instruments. The Super-X detectors have a paired design: detectors 1 and 2 are linked as a pair, whereas detectors 3 and 4 are linked as another pair. Figs. 12b and 12c display the normalized counts as a function of the tilt angle for FEI Talos F200S (equipped with two detectors) and FEI Titan G2 (equipped with four detectors) TEM systems, respectively. The normalized EDS counts for FEI Titan G2 were symmetric. However, the normalized EDS counts for FEI Talos F200S were asymmetric for various tilt angles because the paired detectors were mounted on the same side of the sample holder. Notably, at tilt angles from $+30^{\circ}$ to $+40^{\circ}$, the normalized counts decreased to zero, indicating that a dead angle exists for the two-detector TEM system, suggesting that such a system is not suitable for STEMEDS tomography. Therefore, STEM-EDS tomography can only be performed using a suitable TEM with EDS detectors distributed on both sides of the sample holder.

STEM-EELS tomography is a good alternative to STEM-EDS tomography for the reconstruction of the $3 \mathrm{D}$ distribution of elements or their oxidation states. ${ }^{43}$ A STEM-EELS tomography experiment was performed on a mixture of synthesized np- $\mathrm{Fe}^{0}$ and hematite at a maximum tilt angle of $\pm 54^{\circ}$ and with interval of $6^{\circ}$. The EEL spectra were extracted from the spectral image acquired in the zone shown in Fig. 7a. The $\mathrm{O} K$ - and Fe $L$-edges were identified for hematite, whereas only the $\mathrm{Fe} L$-edge is displayed in the EEL spectra shown in Fig. 7b. From the zoomed-in spectra of the Fe $L$-edges of hematite and np-Fe ${ }^{0}$ (Fig. 7c), the oxidation state of $\mathrm{Fe}$ in both phases could be distinctly identified by the peak positions and shape of the $\mathrm{Fe} L_{3}$-edge (i.e., metallic $\mathrm{Fe}$ at $\sim 708.8$ $\mathrm{eV}$ and $\mathrm{Fe}^{3+}$ at $710.3 \mathrm{eV}$ ). The elemental maps (Figs. 7d-7f) show 
a small amount of oxygen distributed on the surface of np-Fe ${ }^{0}$, suggesting that the surface of $\mathrm{np}-\mathrm{Fe}^{0}$ was slightly oxidized during sample preparation in air. Multiple linear least-squares (MLLS) fitting was conducted to extract the oxidation state maps of iron in the examined zone. The results (Figs. $7 \mathrm{~g}-7 \mathrm{i}$ ) show that $\mathrm{Fe}^{3+}$ was present in the hematite zone and was distributed on the surface of $\mathrm{np}-\mathrm{Fe}^{0}$, further confirming the result acquired from the elemental maps. Furthermore, EELS enables discrimination between $\mathrm{Fe}^{2+}$ and $\mathrm{Fe}^{3+}$, where the $\mathrm{Fe}^{3+} / \sum \mathrm{Fe}$ ratio can be estimated from the $L_{3} / L_{2}$ ratio of the $\mathrm{Fe} L_{2,3}$-edges. The $\mathrm{Fe}^{3+} / \sum \mathrm{Fe}$ ratio could also be used to map the three-dimensional dispersion of iron; the results are consistent with the $\mathrm{Fe}^{3+}$ distribution (Figs. 7h and 9b) in the simple composition (i.e., np- $\mathrm{Fe}^{0}$ and hematite) of the sample and, thus, are not displayed.

Representative slices of the WBP-reconstructed model of np$\mathrm{Fe}^{0}$ and hematite are shown in Fig. 8. The artifacts in the YZ slices of both oxygen and iron in various valences simultaneously show the missing "wedge" from the $\pm 54^{\circ}$ tilt series. Some artifacts were observed on the slices in other directions, similar to the MET results. The features of the slices on the $\mathrm{XZ}$ plane are consistent with those shown in the elemental and oxidation state maps in Fig. 7, suggesting that the reconstructed results successfully reproduced the real features of the examined sample. Furthermore, the $3 \mathrm{D}$ views of the reconstructed models shown in Fig. 9 reveal the actual composition of the specimen.

Atomic electron tomography. AET has been rapidly developing over the past decade. ${ }^{44,45}$ AET can be conducted to determine the $3 \mathrm{D}$ atomic positions in both crystalline and amorphous materials. ${ }^{35}$ This technique may play a critical role in characterizing many noble metals that occur in the lattice of host minerals. Herein, the practicability of AET in resolving the occurrence of gold in the pyrite lattice was verified via theoretical simulations. We first established a cubic pyrite nanoparticle ( $\sim 3 \mathrm{~nm}$ side length, as shown in Fig. 10a) and then simulated a HAADF-STEM tilt series at a maximum tilt angle of $\pm 90^{\circ}$ and with interval of $2^{\circ}$. The tilt series was resampled to extract other tilt series at various maximum tilt angles of $\pm 80^{\circ}, \pm 70^{\circ}, \pm 60^{\circ}$, and $\pm 50^{\circ}$ to demonstrate the effect of the missing "wedge" on the $3 \mathrm{D}$ reconstruction of the atomic positions. Only the simple WBP method was used to perform the reconstruction rather than other advanced algorithms, as employed in previous studies. ${ }^{35,36,46}$ The results (Figs. 10b-10h) show that the positions of iron in the pyrite structure can be easily identified, whereas the sulfur atoms cannot be recognized in the reconstructed slices. The missing "wedge" does not affect the identification of the iron position, but simply distorts the shape of the iron atoms from spherical (Fig. 10g) to fusiform (Fig. 10h).

The gold-bearing pyrite model shown in Fig. 11a was further examined. The HAADF-STEM simulation and reconstruction procedures for the gold-bearing pyrite were similar to those for the pure pyrite model. Based on basic HAADF-STEM theory, the gold atoms in the pyrite lattice can be recognized on the basis of their higher intensity than that of the iron atoms. The maximum tilt angle and interval of the tilt series were considered for reconstruction. The reconstructed results (Figs. 11b-11h) show that neither the maximum tilt angle nor the interval affects the identification of the position of the iron and gold atoms. The maximum tilt angle and interval only affected the atomic shape in the $3 \mathrm{D}$ reconstructions, similar to that observed in the pure pyrite model.

\section{CONCLUSION}

The applicability of several types of ET techniques (MET, SET, and AET) in the earth and planetary science community was verified. MET from a tilt series can allow the reproduction of $3 \mathrm{D}$ spatial relations of objects on the nanoscale level. Both powder and solid samples from the research field of earth and planetary science are suitable for MET. The 3D reconstruction zone should be far from the Pt deposition zone for the FIB-cut samples to avoid carbon contamination. In addition to the 3D morphology, SET can reproduce the $3 \mathrm{D}$ distribution of chemical components and the elemental oxidation state of specimens. Moreover, AET can be conducted to identify the $3 \mathrm{D}$ structure of lattice-bound trace elements; thus, it serves as a promising method for investigating the precise occurrence of critical metals dispersed in host minerals.

\section{AUTHOR INFORMATION}

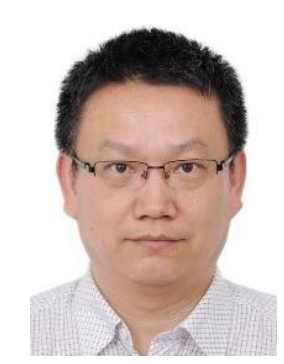

Jianxi Zhu graduated with a B.S degree in Changchun College of Geology in 1996, Ph.D. in mineralogy from University of Chinese Academy of Sciences in 2003. After the postdoctoral research at the Zhejiang University, he joined Guangzhou Institute of Geochemistry, Chinese Academy of Sciences in 2005 and is now professor and director of the Guangdong Provincial Key Laboratory of Mineral Physics and Materials. His research areas include clays and clay minerals, mineral surface chemistry, mineral-based nanomaterials and nano-mineralogy.

\section{Corresponding Author}

*J. X. Zhu

Email address: zhujx@gig.ac.cn

Notes

The authors declare no competing financial interest. 


\section{ACKNOWLEDGMENTS}

This study was financially supported by the National Key R\&D Program of China (2018YFA0702600), Youth Innovation Promotion Association CAS (2021353), Guangdong Basic and Applied Basic Research Foundation (2019A1515011303), Tuguangchi Award for Excellent Young Scholars GIG, CAS, Science and Technology Planning of Guangdong Province, China (2020B1212060055), and Guangdong Special Support Program (2019TX05L169).

\section{REFERENCES}

1. P. Jordan, Z. Phys., 1926, 37, 376-382. https://doi.org/10.1007/Bf01397109

2. D. J. de Rosier and A. Klug, Nature, 1968, 217, 130-134. https://doi.org/10.1038/217130a0

3. P. A. Penczek, Method. Enzymol., 2010, 482, 73-100. https://doi.org/10.1016/S0076-6879(10)82003-8

4. J. Radon, Ber. Verh. K. Sachs. Ges. Wiss. Leipzig, Math.-Phys. Kl., 1917, 69, 262-277.

5. R. N. Bracewell, Aust. J. Phys., 1956, 9, 198-217. https://doi.org/10.1071/PH560198

6. T. J. Marrow, J. Y. Buffiere, P. J. Withers, G. Johnson, and D. Engelberg, Int. J. Fatigue, 2004, 26, 717-725. https://doi.org/10.1016/j.ijfatigue.2003.11.001

7. G. N. Hounsfield, Br. J. Radiol., 1973, 46, 1016-1022. https://doi.org/10.1259/0007-1285-46-552-1016

8. J. Ambrose, Br. J. Radiol., 1973, 46, 1023-1047. https://doi.org/10.1259/0007-1285-46-552-1023

9. K. Baba, K. Satoh, S. Sakamoto, T. Okai, and S. Ishii, J. Perinat. Med., 1989, 17, 19-24. https://doi.org/10.1515/jpme.1989.17.1.19

10. A. Devaraj, D. E. Perea, J. Liu, L. M. Gordon, T. J. Prosa, P. Parikh, D. R. Diercks, S. Meher, R. P. Kolli, Y. S. Meng, and S. Thevuthasan, Int. Mater. Rev., 2018, 63, 68-101. https://doi.org/10.1080/09506608.2016.1270728

11. R. G. Hart, Science, 1968, 159, 1464-1467. https://doi.org/10.1126/science.159.3822.1464

12. W. Hoppe, Optik, 1969, 159, 617-621.

13. R. J. Spontak, M. C. Williams, and D. A. Agard., Polymer, 1988, 29, 387-395. https://doi.org/10.1016/0032-3861(88)90354-0

14. P. Ercius, O. Alaidi, M. J. Rames, and G. Ren, Adv. Mater., 2015, 27, 5638-5663. https://doi.org/10.1002/adma.201501015

15. B. H. Kim, J. Heo, and J. Park, Small Science, 2021, 1, 2000045. https://doi.org/10.1002/smsc.202000045

16. J. H. Li, N. Menguy, E. Leroy, A. P. Roberts, P. Y. Liu, and Y. X. Pan, J. Geophys. Res-Sol Ea, 2020, 125, 1-19. https://doi.org/10.1029/2020JB020853

17. J. Matsuno, A. Tsuchiyama, T. Watanabe, M. Tanaka, A. Takigawa, S. Enju, C. Koike, H. Chihara, and A. Miyake, Astrophys. J., 2021, 911, 47. https://doi.org/10.3847/15384357/abe5a0

18. P. R. Buseck, R. E. Dunin-Borkowski, B. Devouard, R. B. Frankel, M. R. McCartney, P. A. Midgley, M. Posfai, and
M. Weyland, Proc. Natl. Acad. Sci. USA, 2001, 98, 13490-13495. https://doi.org/10.1073/pnas.241387898

19. N. Y. Jin-Phillipp, C. T. Koch, and P. A. van Aken, Ultramicroscopy, 2011, 111, 1255-1261. https://doi.org/10.1016/j.ultramic.2011.02.006

20. G. N. Ramachandran and A. V. Lakshminarayanan, Proc. Natl. Acad. Sci. U S A, 1971, 68, 2236-2240. https://doi.org/10.1073/pnas.68.9.2236

21. J. Frank, Electron Tomography: Three-Dimensional Imaging with the Transmission Electron Microscope. Springer: New York, 1992. https://doi.org/10.1007/978-1-4757-2163-8

22. P. Gilbert, J. Theor. Biol., 1972, 36, 105-117. https://doi.org/10.1016/0022-5193(72)90180-4

23. R. Gordon, R. Bender, and G. T. Herman, J. Theor. Biol., 1970, 29, 471-481. https://doi.org/10.1016/0022-5193(70)90109-8

24. A. Pryor, Jr., Y. S. Yang, A. Rana, M. Gallagher-Jones, J. H. Zhou, Y. H. Lo, G. Melinte, W. Chiu, J. A. Rodriguez, and J. W. Miao, Sci. Rep., 2017, 7, 10409. https://doi.org/10.1038/s41598-017-09847-1

25. C. Y. Wang, G. L. Ding, Y. T. Liu, and H. L. Xin, Advanced Intelligent Systems, 2020, 2, 2000152. https://doi.org/10.1002/aisy.202000152

26. R. A. Crowther, D. J. de Rosier, and A. Klug, Proc. R. Soc. Lond. A., 1970, 317, 319-340.

27. N. Kawase, M. Kato, H. Nishioka, and H. Jinnai, Ultramicroscopy, 2007, 107, 8-15. https://doi.org/10.1016/j.ultramic.2006.04.007

28. M. Radermacher, W. Hoppe, 7th European Congress Electron Microscopy, Den Haag, 1980.

29. C. Suloway, J. Pulokas, D. Fellmann, A. Cheng, F. Guerra, J. Quispe, S. Stagg, C. S. Potter, and B. Carragher, J. Struct. Biol., 2005, 151, 41-60. https://doi.org/10.1016/j.jsb.2005.03.010

30. D. N. Mastronarde, Microsc. Microanal., 2018, 24, 864-865. https://doi.org/10.1017/S1431927618004816

31. D. N. Mastronarde, Microsc. Microanal., 2018, 24, Supplement S1, 864-865. https://doi.org/10.1017/S1431927618004816

32. J. Schindelin, I. Arganda-Carreras, E. Frise, V. Kaynig, M. Longair, T. Pietzsch, S. Preibisch, C. Rueden, S. Saalfeld, B. Schmid, J. Y. Tinevez, D. J. White, V. Hartenstein, K. Eliceiri, P. Tomancak, and A. Cardona, Nat. Methods, 2012, 9, 676-682. https://doi.org/10.1038/nmeth.2019

33. M. D. Hanwell, C. J. Harris, A. Genova, J. Schwartz, Y. Jiang, and R. Hovden, Microsc. Microanal., 2019, 25, 408-409. https://doi.org/10.1017/S1431927619002770

34. D. N. Mastronarde and S. R. Held, J. Struct. Biol., 2017, 197, 102-113. https://doi.org/10.1016/j.jsb.2016.07.011

35. Y. Yang, C.-C. Chen, M. C. Scott, C. Ophus, R. Xu, A. Pryor, L. Wu, F. Sun, W. Theis, J. H. Zhou, M. Eisenbach, P. R. C. Kent, R. F. Sabirianov, H. Zeng, P. Ercius, and J. W. Miao, Nature, 2017, 542, 75-79. https://doi.org/10.1038/nature21042

36. J. Park, H. Elmlund, P. Ercius, J. M. Yuk, D. T. Limmer, Q. Chen, K. Kim, S. H. Han, D. A. Weitz, A. Zettl, and A. P. Alivisatos, Science, 2015, 349, 290-295. https://doi.org/10.1126/science.aab1343

37. M. Merkulova, O. Mathon, P. Glatzel, M. Rovezzi, V. Batanova, P. Marion, M. C. Boiron, and A. Manceau, Acs Earth Space Chem., 2019, 3, 1905-1914. https://doi.org/10.1021/acsearthspacechem.9b00099 
38. J. L. Li, D. M. Feng, Q. Feng, and G. L. Zhang, Acta Geol. Sin. Engl., 2009, 8, 303-315. https://doi.org/10.1111/j.1755-6724.1995.mp8003006.x

39. D. S. He, Z. Y. Li, and J. Yuan, Micron, 2015, 74, 47-53. https://doi.org/10.1016/j.micron.2015.04.005

40. W. Guo, B. T. Sneed, L. Zhou, W. Tang, M. J. Kramer, D. A. Cullen, and J. D. Poplawsky, Microsc. Microanal., 2016, 22, 1251-1260. https://doi.org/10.1017/S1431927616012496

41. S. M. Collins and P. A. Midgley, Ultramicroscopy, 2017, 180, 133-141. https://doi.org/10.1016/j.ultramic.2017.01.003

42. T. J. A. Slater, A. Janssen, P. H. C. Camargo, M. G. Burke, N. J. Zaluzec, and S. J. Haigh, Ultramicroscopy, 2016, 162, 61-73. https://doi.org/10.1016/j.ultramic.2015.10.007
43. K. Jarausch, P. Thomas, D. N. Leonard, R. Twesten, and C. R. Booth, Ultramicroscopy, 2009, 109, 326-337. https://doi.org/10.1016/j.ultramic.2008.12.012

44. J. H. Zhou, Y. Yang, P. Ercius, and J. W. Miao, Mrs Bull., 2020, 45, 290-297. https://doi.org/10.1557/mrs.2020.88

45. J. W. Miao, P. Ercius, and S. J. Billinge, Science, 2016, 353, 2157. https://doi.org/10.1126/science.aaf2157

46. C. C. Chen, C. Zhu, E. R. White, C. Y. Chiu, M. C. Scott, B. C. Regan, L. D. Marks, Y. Huang, and J. W. Miao, Nature, 2013, 496, 74-77. https://doi.org/10.1038/nature12009 